\title{
Prescription du spectre du laplacien de Hodge-de Rham dans une classe conforme
}

Pierre Jammes

Résumé. Sur toute variété compacte de dimension $n \geq 5$, on prescrit le volume et toute partie finie du spectre du laplacien de Hodge-de Rham (sans multiplicité) en restriction aux formes de degré $p \in[2, n-2]$, en excluant $p=n / 2$ si $n$ est pair, et en imposant à la métrique d'appartenir à une classe conforme donnée. On sait que pour $n \leq 4$, ainsi que pour $p=0,1, n-1, n$, et $p=n / 2$ si $n$ est pair, on ne peut pas prescrire simultanément le spectre, le volume et la classe conforme.

\begin{abstract}
For any compact manifold of dimension $n \geq 5$, we prescribe the volume and any finite part of the spectrum of the Hodge Laplacian (without multiplicity) acting on differential forms of degree $p \in[2, n-2]$ (except for $p=n / 2$ if $n$ is even), within a given conformal class. When $n \leq 4$ and when $p=0,1, n-1, n$, and $p=n / 2$ if $n$ is even, this simultaneous prescription of the volume, the spectrum and the conformal class is known to be impossible.
\end{abstract}

Mathematics Subject Classification (2000). 58J50, 58C40, 53A30.

Mots clés. Formes différentielles, laplacien de Hodge-de Rham, prescription de spectre, géométrie conforme.

\section{Introduction}

Étant donnée une suite finie croissante de réels strictement positifs $0<\lambda_{1} \leq \lambda_{2} \leq$ $\cdots \leq \lambda_{k}$, Y. Colin de Verdière a montré dans [CdV87] qu'on peut trouver sur toute variété compacte de dimension supérieure ou égale à 3 une métrique riemannienne telle que le spectre du laplacien agissant sur les fonctions commence par la suite $\left(\lambda_{i}\right)_{i=1}^{k}$, et J. Lohkamp a amélioré ce résultat en montrant dans [Lo96] qu'on pouvait prescrire le volume et certains invariants de courbure en même temps que le spectre. Dans le cas où la suite $\left(\lambda_{i}\right)_{i=1}^{k}$ est strictement croissante, c'est-à-dire en supposant que les valeurs propres prescrites sont simples, des résultats du même type ont été obtenus pour d'autres opérateurs: M. Dahl prescrit dans [Da05] le début du spectre de l'opérateur de Dirac sur les variétés compactes et P. Guérini a montré dans [Gu04] que sur les variétés compactes et les domaines euclidiens, on peut prescrire simultanément 
le volume et toute partie finie du spectre du laplacien de Hodge-de Rham, qui agit sur les formes différentielles.

Notre but est de montrer, dans le cas du laplacien de Hodge-de Rham, que si on munit une variété compacte $M^{n}$ de dimension $n$ d'une métrique riemannienne $g$ quelconque, on peut obtenir le volume et la partie finie du spectre (sans multiplicité) souhaités en effectuant uniquement des déformations conformes à partir de $g$. Il faut noter qu'une telle prescription simultanée du spectre, du volume et de la classe conforme est spécifique aux formes différentielles. Elle est impossible pour le laplacien agissant sur les fonctions: si on fixe le volume et la classe conforme sur une variété compacte donnée, on ne peut pas rendre les valeurs propres arbitrairement grandes ([ESI86], [Ko93]). Sous les mêmes contraintes, on ne peut pas rendre les valeurs propres non nulles de l'opérateur de Dirac arbitrairement petites ([Lo86], [Am03]). Dans le cas des formes différentielles, on se heurte au problème que le spectre des 1 -formes contient le spectre des fonctions, et pour les formes de degré $n / 2$ quand $n$ est pair il y a une obstruction du même type que pour l'opérateur de Dirac (cf. [Ja06], ainsi que la remarque 1.7 ci-dessous). On va montrer qu'on peut prescrire le spectre pour les autres degrés. La comparaison de ces différents résultats permet de mesurer la rigidité qu'apporte le fait de fixer le volume et la classe conforme: on frôle les limites des possibilités de prescription.

Précisons quelques notations: $\mathrm{Si}\left(M^{n}, g\right)$ est une variété riemannienne compacte orientable de dimension $n$, le laplacien $\Delta^{p}$ agissant sur l'espace $\Omega^{p}(M)$ des $p$-formes différentielles est défini par $\Delta=\mathrm{d} \boldsymbol{\delta}+\boldsymbol{\delta} \mathrm{d}$ où $\boldsymbol{\delta}$ désigne la codifférentielle, et son spectre sera noté

$$
0=\lambda_{p, 0}(M, g)<\lambda_{p, 1}(M, g) \leq \lambda_{p, 2}(M, g) \leq \cdots
$$

où les valeurs propres non nulles sont répétées s'il y a multiplicité. La multiplicité de la valeur propre nulle, si elle existe, est un invariant topologique: c'est le nombre de Betti $b_{p}(M)$.

L'espace des $p$-formes coexactes est stable par le laplacien, et on notera

$$
0<\mu_{p, 1}(M, g) \leq \mu_{p, 2}(M, g) \leq \cdots
$$

le spectre du laplacien restreint à cet espace. Par théorie de Hodge, le spectre $\left(\lambda_{p, i}(M, g)\right)_{i \geq 1}$ est la réunion de $\left(\mu_{p, i}(M, g)\right)_{i}$ et $\left(\mu_{p-1, i}(M, g)\right)_{i}$. De plus, si $M$ n'a pas de bord, on a aussi $\mu_{p, i}(M, g)=\mu_{n-p-1, i}(M, g)$ pour tout $p$ et $i$, le spectre complet du laplacien se déduit alors des $\mu_{p, i}(M, g)$ pour $p \leq \frac{n-1}{2}$. Ce sont donc ces valeurs propres qu'on va chercher à prescrire. On exclut le cas $p=0$ puisque $\left(\mu_{0, i}(M, g)\right)$ est le spectre des fonctions, pour lequel la prescription est impossible comme on l'a déjà remarqué. On écarte aussi le cas $p=\left[\frac{n-1}{2}\right]$ pour lequel on ne peut pas rendre les valeurs propres arbitrairement petites (voir remarque 1.7). En particulier, si la dimension de $M$ vérifie $n \leq 4$, la prescription dans une classe conforme et à volume fixé d'une valeur propre quelconque est impossible, quel que soit le degré. 
Compte tenu de ces remarques, en supposant que $n \geq 5$ et en notant $k$ l'entier tel que $n=2 k+3$ ou $2 k+4$, on va prescrire toute partie finie des $\mu_{p, i}(M, g)$ pour $1 \leq p \leq k$.

Théorème 1.3. Soit $M$ une variété compacte, connexe, orientable et sans bord de dimension $n=2 k+3$ ou $2 k+4$ où $k \in \mathbb{N}^{*}, C$ une classe conforme de métriques riemanniennes sur $M, V_{0}$ un réel strictement positif et $N \geq 1$ un entier. On se donne pour tout entier $p \in\{1, \ldots, k\}$ une suite de réels $0<v_{p, 1}<v_{p, 2}<\cdots<v_{p, N}$.

Il existe une métrique $g \in C$ telle que

- $\mu_{p, i}(M, g)=v_{p, i}$ pour tout $i \leq N$ et $p \in\{1, \ldots, k\}$;

- $\mu_{k+1,1}(M, g)>\sup _{p, i}\left\{v_{p, i}\right\}$;

- $\operatorname{Vol}(M, g)=V_{0}$.

Remarque 1.4. La minoration $\mu_{k+1,1}(M, g)>\sup _{p, i}\left\{v_{p, i}\right\}$ assure qu'on a l'égalité $\lambda_{k+1, i}(M, g)=\mu_{k, i}(M, g)$ pour $i \leq N$. On peut donc prescrire les $N$ premières valeurs propres des $(k+1)$-formes, les formes propres correspondantes étant alors exactes, de valeurs propres égales à $\left(\mu_{k, i}(M, g)\right)_{i=1}^{N}$. Si $n$ est impair, on prescrit ainsi le spectre en tout degré $2 \leq p \leq n-2$. En dimension paire, le degré $p=n / 2=k+2$ fait exception. En degré 1 et $n-1$ on ne prescrit pas arbitrairement le début du spectre car on ne contrôle pas les $\mu_{0, i}(M, g)$, mais on peut assurer que les valeurs $v_{1,1}, \ldots, v_{1, N}$ sont contenues dans $\left(\lambda_{1, i}(M, g)\right)_{i \geq 1}$ et $\left(\lambda_{n-1, i}(M, g)\right)_{i \geq 1}$.

Remarque 1.5. Les valeurs propres $\lambda_{p, i}(M, g)$ prescrites sont simples, ou de multiplicité 2 si on fait en sorte que $v_{p, i}=v_{p-1, j}$ pour des valeurs quelconques de $i$ et $j$. Le problème de prescrire arbitrairement la multiplicité ne serait-ce que d'une valeur propre reste à notre connaissance ouvert, tant pour le laplacien de Hodge-de Rham que pour l'opérateur de Dirac.

Une étape clef de la démonstration du théorème 1.3 consiste à montrer que sur la sphère, on peut prescrire une valeur propre, toutes les autres valeurs propres étant arbitrairement grandes, le volume étant majoré et la classe conforme étant fixée. On va montrer un résultat équivalent, à savoir qu'on peut faire tendre une valeur propre non nulle vers zéro en déformant la sphère de manière conforme, les autres valeurs propres étant minorées et le volume étant fixé:

Lemme 1.6. Soit $n \geq 5$ un entier, $k$ l'entier tel que $n=2 k+3$ ou $n=2 k+4$ et $C$ une classe conforme sur $S^{n}$. Pour tout réel $V>0$ et tout entier $1 \leq p \leq k$ il existe une famille de métriques $\left(g_{\varepsilon}\right)_{0<\varepsilon<1}$ contenue dans $C$ et une constante $c>0$ telles que $\mu_{p, 1}\left(S^{n}, g_{\varepsilon}\right)<\varepsilon, \mu_{p, 2}\left(S^{n}, g_{\varepsilon}\right)>c, \mu_{q, 1}\left(S^{n}, g_{\varepsilon}\right)>c$ pour $1 \leq q \leq k+1$, $q \neq p$, et $\operatorname{Vol}\left(S^{n}, g_{\varepsilon}\right)=V$.

Remarque 1.7. Ce lemme ne se généralise pas aux formes différentielles de degré $k+1$; on montre en effet dans [Ja06] qu'une inégalité de Sobolev permet de 
minorer uniformément $\mu_{k+1,1}(M, g) \operatorname{Vol}(M, g)^{2 / n}$ sur une classe conforme par une constante strictement positive. C'est la raison pour laquelle on ne peut pas prescrire $\mu_{k+1, i}(M, g)$ dans le théorème 1.3.

Remarque 1.8. Le problème de faire tendre des valeurs propres vers 0 dans une classe conforme à volume fixé avait été posé par B. Colbois dans [Co04] et était resté ouvert. Le lemme 1.6 y répond partiellement et la technique utilisée permet d'obtenir un grand nombre de petite valeurs propres sur une variété quelconque (voir remarque 2.5 ). Ce problème est totalement résolu par le théorème 1.3 et les résultats de $[\mathrm{Ja} 06]$.

Le lemme 1.6 sera démontré dans la section 2, et le théorème 1.3 dans la section 3 .

Je remercie Bruno Colbois pour de nombreuses discussions autour de ce sujet, ainsi que le rapporteur de l'article dont les remarques ont permis de corriger une erreur et d'améliorer le texte.

\section{Petites valeurs propres dans une classe conforme}

2.1. Quasi-isométries et extrema conformes du spectre. Commençons par rappeler le lemme suivant, dû à J. Dodziuk, qui permet de comparer les spectres de deux métriques dont on connaît le rapport de quasi-isométrie et que nous utiliserons à plusieurs reprises au cours des démonstrations du théorème 1.3 et du lemme 1.6 :

Lemme 2.1 ([Do82]). Soit $g$ et $\tilde{g}$ deux métriques riemanniennes sur une variété compacte $M$ de dimension $n$, et $\tau$ une constante strictement positive. Si les deux métriques vérifient $\frac{1}{\tau} g \leq \tilde{g} \leq \tau g$, alors

$$
\frac{1}{\tau^{3 n-1}} \lambda_{p, k}(M, g) \leq \lambda_{p, k}(M, \tilde{g}) \leq \tau^{3 n-1} \lambda_{p, k}(M, g),
$$

pour tout entiers $k \geq 0$ et $p \in[0, n]$.

Une première conséquence du lemme de Dodziuk est qu'il suffit de démontrer le lemme 1.6 pour une classe conforme particulière, le résultat général s'en déduira : supposons que le lemme soit vrai pour une classe conforme $C$, et donnons-nous une autre classe conforme $C^{\prime}$, ainsi que deux métriques $g \in C$ et $g^{\prime} \in C^{\prime}$, un réel $V>0$ et un entier $p \in[1, k]$. Le lemme 1.6 nous dit que pour tout $\varepsilon>0$ et tout $\tau>1$, il existe une fonction $h \in C^{\infty}(M)$ strictement positive telle que $\mu_{p, 1}\left(M, h^{2} g\right)<\tau^{-1-3 n} \varepsilon$, $\mu_{p, 2}\left(M, h^{2} g\right)>\tau^{3 n+1} c, \mu_{q, 1}\left(M, h^{2} g\right)>\tau^{3 n+1} c$ pour tout $0<p \leq k+1, q \neq p$, et $\operatorname{Vol}\left(M, h^{2} g\right)=V$.

Par compacité de $M$, il existe une constante $\tau>1$ telle que $\frac{1}{\tau} g \leq g^{\prime} \leq \tau g$. Or, les métriques $h^{2} g$ et $h^{2} g^{\prime}$ sont liées par le même rapport de quasi-isométrie que $g$ et $g^{\prime}$, c'est-à-dire que $\frac{1}{\tau} h^{2} g \leq h^{2} g^{\prime} \leq \tau h^{2} g$. On en déduit alors que $\mu_{p, 1}\left(M, h^{2} g^{\prime}\right)<$ 
$\tau^{-2} \varepsilon, \mu_{p, 2}\left(M, h^{2} g^{\prime}\right)>\tau^{2} c, \mu_{q, 1}\left(M, h^{2} g^{\prime}\right)>\tau^{2} c$ pour $q \neq p$ et $\tau^{-n} V \leq$ $\operatorname{Vol}\left(M, h^{2} g\right) \leq \tau^{n} V$. Après renormalisation du volume par homothétie, on a $\mu_{p, 1}\left(M, h^{2} g^{\prime}\right)<\varepsilon, \mu_{p, 2}\left(M, h^{2} g^{\prime}\right)>c, \mu_{q, 1}\left(M, h^{2} g^{\prime}\right)>c$ et $\operatorname{Vol}\left(M, h^{2} g\right)=V$, et donc le lemme 1.6 est vrai pour la classe conforme $C^{\prime}$.

2.2. Construction d'une petite valeur propre sur la sphère. On va maintenant aborder la démonstration du lemme 1.6, qui se déroule en deux étapes. Dans un premier temps on va montrer qu'on peut obtenir une petite valeur propre de degré $p \in[1, k]$ dans une classe conforme donnée et à volume fixé. On vérifiera ensuite que c'est bien la seule petite valeur propre.

Le principe de la construction de cette petite valeur propre est le suivant: on plonge une sphère $S^{p}$ dans $S^{n}$ et on écrase la métrique en dehors d'un voisinage tubulaire de $S^{p}$. On peut alors choisir une forme test qui a un petit quotient de Rayleigh en prolongeant la forme volume de $S^{p}$.

On fixe donc un entier $p \in[1, k]$, et on considère un plongement $i: S^{p} \times B^{n-p} \hookrightarrow$ $S^{n}$ où $B^{n-p}$ est la boule de dimension $n-p$, l'image de $i$ étant un voisinage tubulaire d'une sous-variété de $S^{n}$ difféomorphe à $S^{p}$. Dans la suite, on identifiera $S^{p} \times B^{n-p}$ avec son image par $i$ qu'on notera $\Omega$. On identifiera aussi les formes volumes $\mathrm{d} v_{S^{p}}$ et $\mathrm{d} v_{B^{n-p}}$ de $S^{p}$ et $B^{n-p}$ avec leur relevé sur $\Omega$.

Comme on l'a montré au paragraphe 2.1, il suffit de montrer le lemme pour la classe conforme d'un métrique particulière de $S^{n}$ qu'on choisit comme suit : on muni $B^{n-p}$ d'une métrique euclidienne de rayon $R, S^{p}$ d'une métrique quelconque, $\Omega$ de la métrique produit associée, et $S^{n}$ d'une métrique $g$ qui prolonge la métrique sur $\Omega$. On choisit $R$ suffisamment petit pour qu'on puisse choisir $g$ telle que $\operatorname{Vol}\left(S^{n}, g\right)=$ $V$, et on va montrer qu'on peut trouver une métrique $g_{\varepsilon}$ conforme à $g$ telle que $\mu_{p, 1}\left(S^{n}, g_{\varepsilon}\right)<\varepsilon$ et $\operatorname{Vol}\left(S^{n}, g_{\varepsilon}\right)=V$.

On note $r$ la coordonnée radiale sur $B^{n-p}$ et on se donne une fonction continue $f$ sur $B^{n-p}$ qui ne dépend que de $r$ telle que $f(0)=1, f(R)=0$. On construit une $p$-forme test $\omega$ sur $\Omega$ en posant $\omega=f \mathrm{~d} v_{S^{p}}$, et on l'étend en une $p$-forme sur $S^{n}$ par $\omega=0$ en dehors de $\Omega$. On définit une nouvelle métrique $\bar{g}=h^{2} g$ où $h$ est une autre fonction ne dépendant que de $r$ dans $\Omega$, et constante en dehors. Sur $\Omega$, la forme $\omega$ vérifie $\mathrm{d} \omega=f^{\prime} \mathrm{d} r \wedge \mathrm{d} v_{S}$ et $(-1)^{n(p+1)+1} \delta \omega=* \mathrm{~d}\left(f h^{n-2 p} \mathrm{~d} v_{B}\right)=0$ car $\mathrm{d} r \wedge \mathrm{d} v_{B}=0$.

Précisons le choix de $f$ et de la nouvelle métrique. On veut contracter la métrique dans un domaine de $S^{n}$ qui contient le support de $f^{\prime}$. Étant donné un réel $0<\eta \ll 1$, on choisit la fonction $h$ telle que $h(0)=1, h(1)=\eta$ et qui vaut $\eta$ en dehors de $S^{p} \times B^{n-p}$. On découpe l'intervalle $[0, R]$ en quatre intervalles $I_{i}=\left[\frac{(i-1) R}{4}, \frac{i R}{4}\right]$ et on pose :

- $h(r)=1$ sur $I_{1}$;

- $h(r)=\eta \operatorname{sur} I_{3}, I_{4}$; 
- $f(r)=1$ sur $I_{1}$ et $I_{2}$;

- $f(r)=0$ sur $I_{4}$.

On prolonge $h$ sur $I_{2}$ et $f$ sur $I_{3}$ de manière lisse et monotone (voir figure 1).

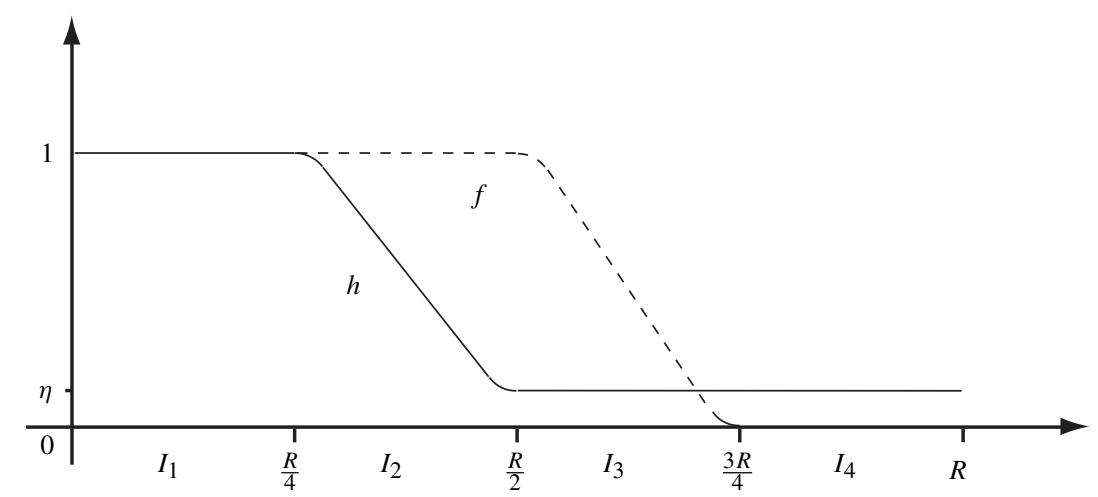

Figure 1

Le quotient de Rayleigh de la forme $\omega$ pour la métrique $\bar{g}$ est

$$
\begin{aligned}
R(\omega) & =\frac{\|\mathrm{d} \omega\|_{\bar{g}}^{2}}{\|\omega\|_{\bar{g}}^{2}}=\frac{\int_{S^{n}} h^{n-2 p-2}|\mathrm{~d} \omega|_{g}^{2} \mathrm{~d} v_{g}}{\int_{S^{n}} h^{n-2 p}|\omega|_{g}^{2} \mathrm{~d} v_{g}} \\
= & \frac{\int_{S^{n}} h^{n-2 p-2}\left|f^{\prime} \mathrm{d} r \wedge \mathrm{d} v_{S^{p}}\right|^{2} \mathrm{~d} v_{g}}{\int_{S^{n}} h^{n-2 p}|f|^{2} \mathrm{~d} v_{g}} .
\end{aligned}
$$

La fonction $f^{\prime}$ est nulle en dehors de $I_{3}$, par conséquent

$$
R(\omega)=\frac{\eta^{n-2 p-2}\left(\int_{r \in I_{3}}\left|f^{\prime}\right|^{2} \mathrm{~d} v_{g}\right)}{\int_{S^{n}} h^{n-2 p}|f|^{2} \mathrm{~d} v_{g}} \leq \eta^{n-2 p-2} \frac{\int_{r \in I_{3}}\left|f^{\prime}\right|^{2} \mathrm{~d} v_{g}}{\int_{r \in I_{1}} \mathrm{~d} v_{g}} .
$$

Dans l'inégalité (2.3), le quotient du membre de gauche est uniformément majoré par rapport à $\eta$. On a supposé que $p \leq k$, donc $n-2 p-2$ est strictement positif. En choisissant $\eta$ suffisamment petit, on obtient une fonction $h_{\varepsilon}$ et une métrique $\bar{g}_{\varepsilon}=h_{\varepsilon}^{2} g$ telle que $R(\omega)<\varepsilon$, et par conséquent $\mu_{p, 1}\left(M, \bar{g}_{\varepsilon}\right)<\varepsilon$ car la forme test est cofermée et la sphère $S^{n}$ n'a pas de cohomologie en degré $p$. Le volume de $S^{n}$ ne fait que 
diminuer lorsque $\eta$ tend vers zéro. Si on le normalise par une homothétie on obtient une métrique $g_{\varepsilon}$ telle que $\operatorname{Vol}\left(S^{n}, g_{\varepsilon}\right)=V$, la petite valeur propre restant inférieure à $\varepsilon$, ce qui conclut la première partie de la démonstration du lemme 1.6.

Remarque 2.4. En dehors du domaines $\Omega$, la métrique ne subit qu'une homothétie. Cette propriété simplifiera la prescription du spectre.

Remarque 2.5. Le procédé que nous venons de décrire se généralise aisément pour construire un grand nombre de petites valeurs propres pour plusieurs degrés simultanément sur une variété compacte $M$ quelconque : à partir d'un nombre arbitraire de sphères plongées dans $M$, on peut construire autant de formes test dont le quotient de Rayleigh tend vers zéro quand on écrase la métrique en dehors de voisinages tubulaires tous disjoints de ces sphères. Cependant, si la variété a des nombres de Betti non nuls, on ne contrôle pas précisément le nombre de petites valeurs propres non nulles obtenu.

2.3. Contrôle du nombre de petites valeurs propres. Pour achever la démonstration du lemme 1.6, on doit s'assurer que la petite valeur propre construite au paragraphe précédent est bien la seule. Nous allons pour cela faire appel à un lemme dû à J. McGowan qui permet, étant donné un recouvrement de la variété par des ouverts à bords lisses, de minorer une partie du spectre de la variété en fonction du spectre des ouverts du recouvrement et de leurs intersections. Sur les domaines de la variété, le spectre que nous considérerons sera toujours celui du laplacien agissant sur les formes vérifiant la condition de bord absolue, qui généralise la condition de Neumann, à savoir :

$$
\left\{\begin{array}{l}
j^{*} \mathbf{\iota}_{v} \omega=0 \\
j^{*} \mathbf{\iota}_{\nu} \mathrm{d} \omega=0,
\end{array}\right.
$$

où $j: \partial M \hookrightarrow M$ désigne l'inclusion canonique, $v$ la normale au bord et $\mathbf{t}_{v}$ le produit intérieur par $v$.

Lemme 2.7. Soit $(M, g)$ une variété compacte de dimension $n,\left(U_{i}\right)_{i=1}^{K}$ un recouvrement de $M$ par des ouverts n'ayant pas d'intersections d'ordre supérieur ou égal à 3 et $\rho_{i}$ une partition de l'unité relative à $\left(U_{i}\right)_{i=1}^{K}$. Il existe des constantes $a, b>0$ ne dépendant que de $n$ telles que

$$
\mu_{q, k_{q}}(M, g) \geq \frac{a}{\sum_{i=1}^{K}\left(\frac{1}{\mu_{i}}+\sum_{U_{i} \cap U_{j} \neq \emptyset}\left(\frac{b \cdot c_{\rho}}{\mu_{i j}}+1\right)\left(\frac{1}{\mu_{i}}+\frac{1}{\mu_{j}}\right)\right)}
$$

avec $k_{q}=1+\sum_{i, j} \operatorname{dim} \mathscr{H}^{q}\left(\bar{U}_{i} \cap \bar{U}_{j}\right)$ où $\mathscr{H}^{q}$ désigne l'espace des $q$-formes harmoniques avec condition de bord absolue, $\mu_{i}=\mu_{q, 1}\left(\bar{U}_{i}\right), \mu_{i j}=\mu_{q-1,1}\left(\bar{U}_{i} \cap \bar{U}_{j}\right)$ et $c_{\rho}=\sup _{i}\left\|\nabla \rho_{i}\right\|_{\infty}^{2}$. 
Ce lemme a été démontré dans [Mc93] pour les 1-formes pour un recouvrement fini quelconque, et G. Gentile et V. Pagliara ont remarqué dans [GP95] qu'il se généralise aux formes de degré quelconque si on suppose que les ouverts du recouvrement n'ont pas d'intersection d'ordre supérieur ou égal à 3 .

Nous allons appliquer ce lemme au recouvrement constitué des deux ouverts $U_{1}$ formé de l'intérieur de $\Omega$ et $U_{2}$ formé de $S^{n}$ privé des points de $\Omega$ tels que $r \leq \frac{3 R}{4}$. Par commodité, on appliquera le lemme 2.7 pour la métrique $\bar{g}_{\varepsilon}$ au lieu de $g_{\varepsilon}$. Comme $\operatorname{Vol}\left(S^{n}, \bar{g}_{\varepsilon}\right)$ reste uniformément minoré quand $\varepsilon \rightarrow 0$, cela ne change pas significativement le résultat.

Le nombre $k_{q}$ du lemme 2.7 vaut 2 pour $q=p$ et 1 pour les autres degrés $q \leq k+1$. Par conséquent, la minoration des termes $\mu_{1}, \mu_{2}$ et $\frac{\mu_{12}}{c_{\rho}}$ du lemme 2.7 permettrait de conclure la démonstration.

On peut noter qu'au cours de la déformation conforme créant la petite valeur propre, l'intersection $U_{1} \cap U_{2}$ ne subit qu' une homothétie. En choisissant une partition de l'unité indépendante de $\bar{g}_{\varepsilon}$, le rapport $\frac{c_{\rho}}{\mu_{12}}$ dans le lemme 2.7 est invariant par homothétie, il est donc indépendant de $\varepsilon$. Par ailleurs, la métrique sur $U_{2}$ subit une homothétie qui fait tendre son spectre non nul vers l'infini.

Pour minorer $\mu_{q, k_{q}}\left(S^{n}, \bar{g}_{\varepsilon}\right)$ à l'aide du lemme 2.7 il reste donc à minorer le spectre de $U_{1}$, pour lequel une difficulté apparaît : la métrique sur ce domaine n'est pas fixe puisqu'on l'écrase sur un voisinage du bord, et il n'est pas clair a priori que cette déformation ne produise pas de petites valeurs propres : on a vu précédemment que si une telle déformation se produit ailleurs que près du bord, elle peut effectivement faire tendre une valeur propre vers zéro.

Fait 2.8. Il existe une constante $c>0$ indépendante du choix de $h_{\varepsilon}$ telle que $\mu_{q, 1}\left(\Omega, h_{\varepsilon}^{2} g\right)>c$, pour tout $1 \leq q \leq k+1$.

Démonstration. On utilise une nouvelle fois le lemme de Dodziuk pour se ramener à une classe conforme particulière en identifiant $B^{n-p}$ à la réunion d'un cylindre $[0,1] \times S^{n-p-1}$ (muni d'une métrique produit) et d'un hémisphère (voir figure 2), en munissant $S^{p}$ de sa métrique canonique et $\Omega$ de la métrique produit.

On recouvre $\Omega$ par deux domaines, $\Omega_{1}$ défini comme le produit de $S^{p}$ et de réunion de l'hémisphère et de $\left[0, \frac{1}{4}\right] \times S^{n-p-1}$, et $\Omega_{2}$ formé de $[0,1] \times S^{n-p-1} \times S^{p}$. On va minorer le spectre sur chacun des deux domaines et en déduire une minoration sur $\Omega$. On ne peut pas appliquer le lemme de McGowan pour minorer la totalité du spectre non nul car la cohomologie des intersections des domaines est non triviale, mais dans cette situation on peut en adapter la démonstration pour obtenir un résultat plus précis.

Remarquons d'abord que la métrique sur $\Omega_{1}$ est fixe, son spectre est donc uniformément minoré par rapport à $\varepsilon$. On doit ensuite minorer le spectre de $\Omega_{2}$. Sur ce domaine, la métrique $g$ est le produit des métriques canoniques de $[0,1], S^{p}$ et 
$S^{n-p-1}$. Elle est donc invariante sous l'action des groupes d'isométries $\mathrm{SO}(p+1)$ et $\mathrm{SO}(n-p)$ des sphères $S^{p}$ et $S^{n-p-1}$, et il en est de même pour la métrique $h_{\varepsilon}^{2} g$. Or, on peut montrer (voir [Ja04], théorème 1.18 et section 4) que lorsque le cercle agit par isométrie sur une variété compacte, il existe une constante dépendant uniquement de la longueur maximale des orbites telle que si une valeur propre est inférieure à cette constante, les formes propres correspondantes sont invariantes sous l'action du cercle. Le groupe $\mathrm{SO}(p+1) \times \mathrm{SO}(n-p)$ étant engendré par des cercles dont la longueur des orbites est uniformément majorée par rapport à $\varepsilon$, on peut se restreindre aux formes invariantes par cette action. Remarquons en outre que les seules formes invariantes de la sphère sont les fonctions constantes et les multiples de la forme volume canonique.

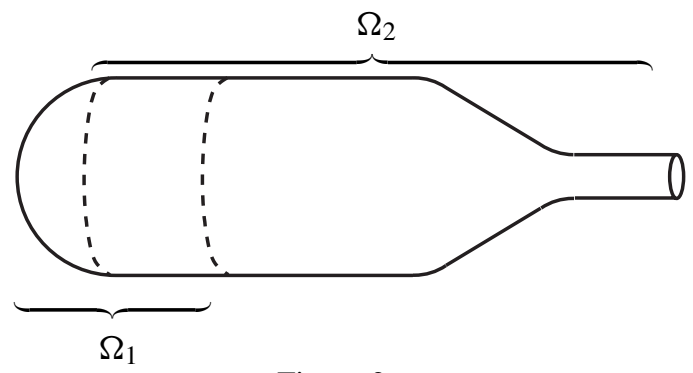

Figure 2

Ces remarques nous permettent de nous ramener à un problème unidimensionnel: une forme propre de $\left(\Omega_{2}, h^{2} g\right)$ de degré compris entre 1 et $k+1$ et dont la valeur propre est petite est nécessairement de la forme

$$
\omega=f(t) \mathrm{d} v_{S^{p}} \text { ou } \omega=f(t) \mathrm{d} v_{S^{p}} \wedge \mathrm{d} t,
$$

où $f$ est une fonction sur $[0,1]$ et $\mathrm{d} v_{S^{p}}$ la forme volume de $S^{p}$, qu'on identifie avec leur relevé à $\Omega_{2}$. L'image de $\omega$ par d étant aussi invariante, on peut affirmer que $f(t) \mathrm{d} v_{S^{p}} \wedge \mathrm{d} t$ est fermée. Comme il suffit de minorer le spectre des formes coexactes, on va raisonner sur $\omega=f(t) \mathrm{d} v_{S^{p}}$.

On va calculer explicitement le laplacien de $\omega$ en fonction de $f$ et $h$, puis en déduire que $\omega$ ne peut pas être une forme propre de valeur propre petite non nulle. Remarquons d'abord que $\mathrm{d} * \omega=\mathrm{d}\left(h(t)^{n-2 p} f(t) \mathrm{d} v_{S^{n-p-1}} \wedge \mathrm{d} t\right)=0$, c'est-à-dire que $\delta \omega=0$. On a par ailleurs $\mathrm{d} \omega=f^{\prime}(t) \mathrm{d} t \wedge \mathrm{d} v_{S^{p}}$, donc

$$
\begin{aligned}
\mathrm{d} * \mathrm{~d} \omega & =(-1)^{n-1} \mathrm{~d}\left(f^{\prime} h^{n-2 p-2}\right) \mathrm{d} v_{S^{n-p-1}} \\
& =(-1)^{p}\left[h^{n-2 p-2} f^{\prime \prime}+(n-2 p-2) f^{\prime} h^{\prime} h^{n-2 p-3}\right] \mathrm{d} v_{S^{n-p-1}} \wedge \mathrm{d} t
\end{aligned}
$$

et finalement

$$
\Delta \omega=\delta \mathrm{d} \omega=-\left[h^{-2} f^{\prime \prime}+(n-2 p-2) f^{\prime} h^{\prime} h^{-3}\right] \mathrm{d} v_{S^{p}} .
$$


Le même calcul montre que sur la variété $S^{n-2 p-1} \times[0,1]$ munie de la métrique $h^{2} g^{\prime}$ où $g^{\prime}$ est la métrique produit, la fonction $f$ vérifie $\Delta f=-\left(h^{-2} f^{\prime \prime}+(n-\right.$ $2 p-2) f^{\prime} h^{\prime} h^{-3}$ ). Si $\omega$ est une forme propre de valeur propre $\lambda>0$, alors $f$ est une fonction propre sur $\left(S^{n-2 p-1} \times[0,1], h^{2} g^{\prime}\right)$ de même valeur propre. Or, si on choisit la famille $h_{\varepsilon}$ de sorte qu'elle tende simplement vers la fonction caractéristique de $[0,1 / 2]$, on sait que cette déformation ne produit pas de petites valeurs propres non nulles pour les fonctions (c'est un corollaire immédiat du théorème III.3 de [CdV86]). Le spectre non nul de $\Omega_{2}$ est donc bien uniformément minoré.

On doit maintenant déduire une minoration du spectre de $\Omega$ des minorations des spectres de $\Omega_{1}$ et $\Omega_{2}$. Pour les degrés autres que $p$ on peut appliquer le lemme 2.7. En degré $p$ ce lemme ne minore que la $2^{\mathrm{e}}$ valeur propre, on va voir comment améliorer ce résultat. On se référera implicitement au début de la section 2 de l'article [Mc93] de J. McGowan pour les notions de théorie de Hodge et de théorie spectrale des variétés à bord que nous utiliserons. Rappelons-en deux points techniques: tout d'abord, comme l'a expliqué J. Dodziuk dans [Do82], il suffit pour minorer $\mu_{p, 1}$ de trouver une constante $C>0$ telle que pour toute $(p+1)$-forme exacte $\phi$, il existe une $p$ forme $\psi$ telle que $\mathrm{d} \psi=\phi$ et $\|\psi\| /\|\phi\| \leq C$, et par ailleurs, à l'exception des formes harmoniques, on a pas besoin de se restreindre aux formes différentielles vérifiant les conditions de bord.

Soit $\phi$ une $(p+1)$-forme exacte sur $\Omega$. Pour tout $i=1,2$, on note $\phi_{i}$ sa restriction au domaine $\Omega_{i}$. Chaque $\phi_{i}$ est une forme exacte, donc il existe sur chacun des $\Omega_{i}$ une forme $\psi_{i}$ telle que $\mathrm{d} \psi_{i}=\phi_{i}$, et on peut choisir $\psi_{i}$ telle que $\mu_{p, 1}\left(\Omega_{i}\right)\left\|\psi_{i}\right\|^{2} \leq\left\|\phi_{i}\right\|^{2}$.

Sur l'intersection $\Omega_{12}=\Omega_{1} \cap \Omega_{2}$, on peut définir la forme $\omega=\psi_{2}-\psi_{1}$. Elle vérifie $\mathrm{d} \omega=\mathrm{d} \psi_{2}-\mathrm{d} \psi_{1}=0$, on peut donc l'écrire sous la forme $\omega=\alpha+\mathrm{d} \beta$, où $\alpha$ est une $p$-forme harmonique - avec conditions de bord absolues - de $\Omega_{12} \simeq$ $S^{p} \times S^{n-p-1} \times\left[0, \frac{1}{4}\right]$ et qui est donc nécessairement proportionnelle au relevé de $\mathrm{d} v_{S^{p}}$. Cette forme s'étend naturellement en une forme harmonique (pour la métrique g) de $\Omega_{2} \simeq S^{p} \times S^{n-p-1} \times[0,1]$, qui est de longueur constante pour $g$ et qu'on notera encore $\alpha$.

Soit $\left(\rho_{i}\right)$ une partition de l'unité pour le recouvrement $\left(\Omega_{i}\right)$. La forme $\rho_{1} \beta$ (resp. $\rho_{2} \beta$ ) qui est définie sur $\Omega_{12}$ se prolonge naturellement par 0 en dehors de $\Omega_{12}$ pour donner une forme sur $\Omega_{2}$ (resp. $\Omega_{1}$ ). On définit alors sur les domaines $\Omega_{i}$ les formes $\bar{\psi}_{i}$ par

$$
\bar{\psi}_{1}=\psi_{1}+\mathrm{d}\left(\rho_{2} \beta\right) \text { et } \bar{\psi}_{2}=\psi_{2}-\alpha-\mathrm{d}\left(\rho_{1} \beta\right) .
$$

Ces formes vérifient $\mathrm{d} \bar{\psi}_{i}=\mathrm{d} \psi_{i}=\phi_{i}$ pour $i=1,2$, et sur $\Omega_{12}$, on a

$$
\bar{\psi}_{2}-\bar{\psi}_{1}=\psi_{2}-\psi_{1}-\alpha-\mathrm{d}\left(\left(\rho_{1}+\rho_{2}\right) \beta\right)=\omega-\alpha-\mathrm{d} \beta=0 .
$$

Les formes $\left(\bar{\psi}_{i}\right)_{i=1,2}$ coïncident sur l'intersection $\Omega_{12}$, ce sont donc les restrictions d'une forme globale sur $\Omega$ qu'on notera $\psi$, et qui vérifie $\mathrm{d} \psi=\phi$. Pour minorer le spectre de $\Omega$, on doit majorer la norme de $\psi$ en fonction de celle de $\phi$, indépendam- 
ment du choix de $h_{\varepsilon}$. On commence par écrire

$$
\begin{aligned}
\|\psi\|^{2} & \leq\left\|\bar{\psi}_{1}\right\|^{2}+\left\|\bar{\psi}_{2}\right\|^{2} \leq\left\|\psi_{1}+\mathrm{d}\left(\rho_{2} \beta\right)\right\|^{2}+\left\|\psi_{2}-\alpha-\mathrm{d}\left(\rho_{1} \beta\right)\right\|^{2} \\
& \leq 3\left(\left\|\psi_{1}\right\|^{2}+\left\|\psi_{2}\right\|^{2}+\left\|\mathrm{d}\left(\rho_{1} \beta\right)\right\|^{2}+\left\|\mathrm{d}\left(\rho_{2} \beta\right)\right\|^{2}+\|\alpha\|^{2}\right) .
\end{aligned}
$$

Dans l'inégalité précédente, chaque norme considérée est relative au domaine $\Omega_{i}$ sur lequel la forme est définie. On peut majorer les premiers termes en utilisant le fait que $\mu_{p, 1}\left(\Omega_{i}\right)\left\|\psi_{i}\right\|^{2} \leq\left\|\phi_{i}\right\|^{2} \leq\|\phi\|^{2}$. Pour les termes faisant intervenir $\alpha$ et $\beta$, on se ramène aux normes sur $\Omega_{12}$ : comme les supports des formes $\rho_{i} \beta$ sont contenus dans $\Omega_{12}$, leur norme ne change pas quand on se restreint à $\Omega_{12}$, et en utilisant le le fait que $\alpha$ est de degré $p$ inférieur à $k$ et qu'elle est de longueur constante pour la métrique $g$ on a

$$
\|\alpha\|_{\Omega_{2}, h_{\varepsilon}^{2} g} \leq\|\alpha\|_{\Omega_{2}, g} \leq \frac{\operatorname{Vol}\left(\Omega_{2}, g\right)}{\operatorname{Vol}\left(\Omega_{12}\right)}\|\alpha\|_{\Omega_{12}} .
$$

On peut alors majorer les termes restants de l'inégalité (2.14) en commençant par

$$
\frac{1}{2}\left\|\mathrm{~d}\left(\rho_{i} \beta\right)\right\|^{2} \leq c_{\rho}\|\beta\|^{2}+\|\mathrm{d} \beta\|^{2} \leq\|\mathrm{d} \beta\|^{2}\left(\frac{c_{\rho}}{\mu_{p-1,1}\left(\Omega_{12}\right)}+1\right),
$$

où $c_{\rho}=\sup _{i}\left\|\nabla \rho_{i}\right\|_{\infty}^{2}$. On majore les normes de $\alpha$ et $\mathrm{d} \beta$ en partant de l'égalité $\alpha+\mathrm{d} \beta=\psi_{2}-\psi_{1}$ et en utilisant le fait que $\alpha$ est harmonique avec condition de bord absolue, donc orthogonale aux formes exactes, ce qui donne

$$
\begin{aligned}
\|\mathrm{d} \beta\|^{2} & \leq\left\|\psi_{2}-\psi_{1}\right\|^{2} \leq 2\left\|\psi_{1}\right\|^{2}+2\left\|\psi_{2}\right\|^{2} \\
& \leq\left(\frac{2}{\mu_{p, 1}\left(\Omega_{1}\right)}+\frac{2}{\mu_{p, 1}\left(\Omega_{2}\right)}\right)\|\phi\|^{2}
\end{aligned}
$$

et de la même manière,

$$
\|\alpha\|^{2} \leq\left(\frac{2}{\mu_{p, 1}\left(\Omega_{1}\right)}+\frac{2}{\mu_{p, 1}\left(\Omega_{2}\right)}\right)\|\phi\|^{2} .
$$

On a donc une majoration du quotient $\|\psi\|^{2} /\|\phi\|^{2}$ en fonction de $c_{\rho}$, de $\mu_{p-1,1}\left(\Omega_{12}\right)$, des $\left(\mu_{p, 1}\left(\Omega_{i}\right)\right)_{i=1,2}$ et du rapport $\frac{\operatorname{Vol}\left(\Omega_{2}, g\right)}{\operatorname{Vol}\left(\Omega_{12}\right)}$. Seuls $\mu_{p, 1}\left(\Omega_{2}\right)$ et $\frac{\operatorname{Vol}\left(\Omega_{2}, g\right)}{\operatorname{Vol}\left(\Omega_{12}\right)}$ dépendent du choix de $h_{\varepsilon}$, et ils sont uniformément minorés.

On a finalement une minoration uniforme de $\mu_{p, 2}\left(M, g_{\varepsilon}\right)$ et $\mu_{q, 1}\left(M, g_{\varepsilon}\right)$ pour $1 \leq q \leq k+1, q \neq p$, l'application du lemme 2.7 permet donc de conclure la démonstration du lemme 1.6. 


\section{Prescription du spectre}

3.1. Propriétés de convergence et de stabilité du spectre. Pour prescrire le spectre dans classe conforme, nous allons utiliser des techniques déjà mises en œuvre par P. Guérini dans [Gu04] et que nous allons rappeler ici. Nous expliquerons ensuite comment la construction géométrique considérée peut être réalisée de manière conforme à partir d'une métrique quelconque.

Un premier outil est le résultat de convergence de spectre obtenu par C. Anné et B. Colbois dans [AC95] pour les variétés compactes reliées par des anses fines. Considérons une famille finie de variétés compactes $\left(M_{j}, g_{j}\right)_{i=1}^{K}$ qu'on relie entre elles par des anses fines, isométriques au produit d'une sphère $\left(S^{n-1}, \varepsilon^{2} g_{\text {can }}\right)$ par un intervalle (voir figure 3$)$. En notant $\left(\tilde{M}, g_{\varepsilon}\right)$ la variété obtenue, qui est difféomorphe à $M_{1} \# M_{2} \# \cdots \# M_{K}$, on a alors :

Théorème 3.1. Si, pour $p \in\{1, \cdots, n-1\}$, on note $\mu_{p, 1}^{\prime} \leq \mu_{p, 2}^{\prime} \leq \cdots$ la réunion des spectres $\left(\mu_{p, i}\left(M_{j}, g_{j}\right)\right)_{i, j}$, on a pour tout $i \in \mathbb{N}^{*}$

$$
\lim _{\varepsilon \rightarrow 0} \mu_{p, i}\left(\tilde{M}, g_{\varepsilon}\right)=\mu_{p, i}^{\prime}
$$

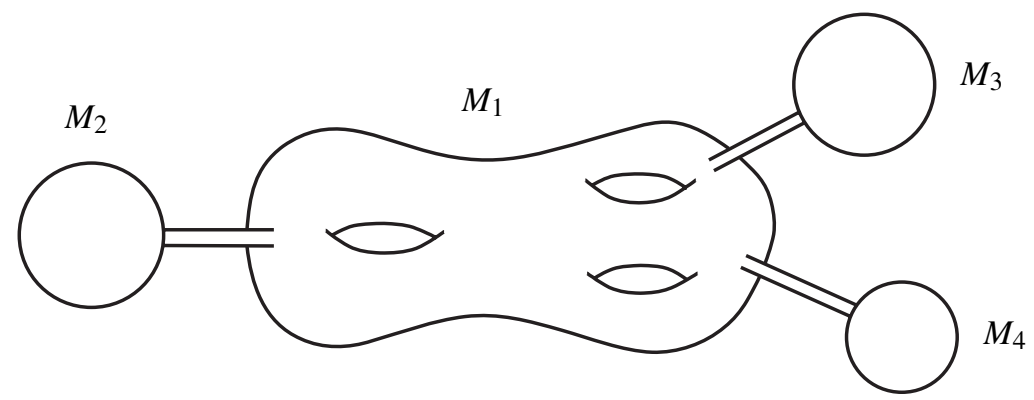

Figure 3

Décrivons rapidement comment prescrire le spectre (les détails seront précisés au paragraphe suivant): on commence par fixer un réel strictement positif $\delta$ tel que

$$
\delta<\inf _{p \leq k, i<N}\left\{\frac{v_{p, i+1}-v_{p, i}}{2}\right\} \text { et } \delta<V_{0}
$$

et choisir un réel $V \in\left[V_{0}-\delta, V_{0}+\delta\right]$.

Pour tout $1 \leq p \leq k$ et tout $1 \leq i \leq N$, on se donne un réel $\xi_{p, i} \in\left[v_{p, i}-\right.$ $\delta, v_{p, i}+\delta$ ] et une métrique $g_{p, i}$ sur la sphère $S^{n}$ telle que $\operatorname{Vol}\left(S^{n}, g_{p, i}\right)<\frac{V}{N k}$ et $\mu_{p, 1}\left(S^{n}, g_{p, i}\right)=\xi_{p, i}$, toutes les autres valeurs propres de la sphère étant supérieures 
à $\sup _{p, i}\left\{v_{p, i}\right\}+\delta$. On munit $M$ d'une métrique telle que $\mu_{p, 1}(M)>\sup _{p, i}\left\{v_{p, i}\right\}+\delta$ pour tout $p$ compris entre 1 et $n-2$, et $\operatorname{Vol}(M)=V-\sum_{p, i} \operatorname{Vol}\left(S^{n}, g_{p, i}\right)$. Si on attache les sphères à $M$ par des anses fines comme dans la figure 3 , on obtient une variété difféomorphe à $M$, et le théorème 3.1 nous donne alors une famille de métrique $\left(g_{\varepsilon}\right)$ sur $M$ telle que $\operatorname{Vol}\left(M, g_{\varepsilon}\right)$ tend vers $V$ et $\mu_{p, i}\left(M, g_{\varepsilon}\right)$ tend vers $\xi_{p, i}$ pour tout $p$ et $i$ quand $\varepsilon$ tend vers zéro. Les métriques $g_{\varepsilon}$ sont singulières sur le bord des anses, mais on verra qu' on peut les lisser sans perturber la convergence du spectre.

On peut alors utiliser l'argument de stabilité développé par Y. Colin de Verdière dans [CdV86]. On s'appuie sur le

Lemme 3.3. Soit $\left(\Phi_{\varepsilon}\right)_{\varepsilon}$ une famille d'applications continues d'une boule fermée $B_{0} \subset \mathbb{R}^{m}$ dans $\mathbb{R}^{m}$ qui converge uniformément vers l'identité quand $\varepsilon$ tend vers 0 , et $x_{0}$ un point intérieur à $B_{0}$.

Si $\varepsilon$ est suffisamment petit, alors $x_{0}$ est contenu dans l'image de $\Phi_{\varepsilon}$.

On applique ce lemme à l'espace $\mathbb{R}^{k N+1}$ avec

$$
\begin{aligned}
\Phi_{\varepsilon}:\left[V_{0}-\delta, V_{0}+\delta\right] \times \prod_{p, i}\left[v_{p, i}-\delta, v_{p, i}+\delta\right] & \rightarrow \mathbb{R}^{k N+1} \\
\left(V, \xi_{1,1}, \ldots, \xi_{1, N}, \ldots, \xi_{k, 1}, \ldots, \xi_{k, N}\right) & \mapsto\left(\operatorname{Vol}\left(M, g_{\varepsilon}\right), \mu_{p, i}\left(M, g_{\varepsilon}\right)\right)
\end{aligned}
$$

et $x_{0}=\left(V_{0}, v_{1,1} \ldots, v_{1, N}, v_{k, 1}, \ldots, v_{k, N}\right)$. Selon le théorème 3.1, $\Phi_{\varepsilon}$ converge simplement vers l'identité quand $\varepsilon \rightarrow 0$. Mais comme ces applications sont continues et que $B_{0}$ est compact, le théorème de Dini garantit que la convergence est uniforme. On peut donc trouver un jeu de paramètres $\left(V, \xi_{i, j}\right)$ et un $\varepsilon$ telle que la métrique $g_{\varepsilon}$ vérifie les conclusions du théorème. Comme $\mu_{k+1,1}\left(M, g_{\varepsilon}\right)$ tend vers une valeur supérieure à $\sup _{p, i}\left\{v_{p, i}\right\}+\delta$, on peut choisir $\varepsilon$ suffisamment petit pour que la condition $\mu_{k+1,1}(M, g)>\sup _{p, i}\left\{v_{p, i}\right\}$ soit vérifiée.

Notons qu'il est est essentiel que pour chaque valeur de $p$ les termes de la suite $\left(v_{p, i}\right)_{i=1}^{N}$ soient distincts, afin qu'on puisse choisir $B_{0}$ et $x_{0}$ tels que $x_{0}$ soit à l'intérieur de $B_{0}$.

3.2. Prescription conforme. Il reste à montrer que la construction précédente peut être réalisée de manière conforme.

La première étape consiste, pour chaque $\xi_{p_{0}, i}$, à trouver la métrique $g_{p_{0}, i}$ correspondante sur la sphère. Pour cela, on applique le lemme 1.6 en considérant la classe conforme de la métrique canonique, et

$$
\varepsilon<\frac{c \cdot \xi_{p_{0}, i}}{\sup _{p, i}\left\{v_{p, i}\right\}+\delta}
$$


On obtient une métrique $\bar{g}_{p_{0}, i}$ telle que $\mu_{p_{0}, 1}\left(S^{n}, \bar{g}_{p_{0}, i}\right)<\varepsilon$, les autres valeurs propres étant supérieures à $c$. En posant

$$
g_{p_{0}, i}=\frac{\mu_{p_{0}, 1}\left(S^{n}, \bar{g}_{p_{0}, i}\right)}{\xi_{p_{0}, i}} \bar{g}_{p_{0}, i}
$$

on a $\mu_{p_{0}, 1}\left(S^{n}, g_{p_{0}, i}\right)=\xi_{p_{0}, i}$, et les autres valeurs propres sont supérieures à $c$. $\frac{\xi_{p_{0}, i}}{\mu_{p_{0}, 1}\left(S^{n}, \bar{g}_{p_{0}, i}\right)}>c \cdot \frac{\xi_{p_{0}, i}}{\varepsilon}>\sup _{p, i}\left\{v_{p, i}\right\}+\delta$. Le lemme 1.6 permet en outre de majorer le volume de la sphère pour la métrique $\bar{g}_{p_{0}, i}$, et donc pour la métrique $g_{p_{0}, i}$. On peut donc choisir $g_{p_{0}, i}$ telle que $\operatorname{Vol}\left(S^{n}, g_{p_{0}, i}\right)<\frac{V}{N k}$.

On veut ensuite munir $M$ d'une métrique pour laquelle toutes les valeurs propres sont grandes, le volume et la classe conforme étant fixés. C'est possible en vertu d'un résultat de B. Colbois et A. El Soufi:

Théorème 3.7 ([CES06]). Si $M$ est une variété riemannienne compacte de dimension $n \geq 4$ et $C$ une classe conforme sur $M$, alors

$$
\sup _{g \in C} \inf _{0<p<n-2} \mu_{p, 1}(M, g) \operatorname{Vol}(M, g)^{\frac{2}{n}}=+\infty .
$$

On peut donc se donner une métrique $g$ sur $M$ telle que

$$
\operatorname{Vol}(M, g)=V-\sum_{p_{0}, i} \operatorname{Vol}\left(S^{n}, g_{p_{0}, i}\right)
$$

et

$$
\mu_{p_{0}, 1}(M, g)>\sup _{p, i}\left\{v_{p, i}\right\}+\delta
$$

pour tout $0<p_{0}<n-1$.

On veut maintenant attacher des sphères à $M$ par des anses fines. Un réel $\varepsilon>0$ petit étant donné, on va - temporairement - déformer la métrique $g$ de manière non conforme en une métrique $g_{\varepsilon}$ telle que cette métrique soit euclidienne sur $k N$ boules disjointes de rayon $\varepsilon$. On peut choisir $g_{\varepsilon}$ telle que $\frac{1}{\tau(\varepsilon)} g \leq g_{\varepsilon} \leq \tau(\varepsilon) g$, avec $\tau(\varepsilon) \rightarrow 1$ quand $\varepsilon \rightarrow 0$. Comme l'ont remarqué B. Colbois et A. El Soufi dans [CES03] et [CES06], une boule euclidienne $\left(B(\varepsilon), g_{\text {euc }}\right)$ de rayon $\varepsilon$ peut être déformée de manière conforme en la réunion d'un cylindre de rayon $\varepsilon$ et de longueur quelconque, et d'une sphère homothétique à la sphère canonique privée d'une boule de rayon $\varepsilon$ : si on note $r$ le coordonnée radiale sur $B(\varepsilon)$ et qu'on définit la fonction $h_{1, \varepsilon}$ par

$$
h_{1, \varepsilon}(r)= \begin{cases}\frac{\varepsilon}{r} & \text { si } \varepsilon e^{-\frac{L}{\varepsilon}} \leq r \leq \varepsilon, \\ e^{\frac{L}{\varepsilon}} & \text { si } 0 \leq r \leq \varepsilon e^{-\frac{L}{\varepsilon}},\end{cases}
$$


Une fois munie de la métrique $h_{1, \varepsilon}^{2} g_{\text {euc }}$, la partie de la boule $B(\varepsilon)$ correspondant à $r \in\left[\varepsilon e^{-\frac{L}{\varepsilon}}, \varepsilon\right]$ est isométrique à un cylindre de longueur $L$ et de rayon $\varepsilon$ tandis que la partie correspondant à $r \in\left[0, \varepsilon e^{-\frac{L}{\varepsilon}}\right]$ est isométrique à une boule euclidienne de rayon $\varepsilon$. Comme cette boule peut être projetée stéréographiquement - donc de manière conforme - sur une calotte sphérique quelconque, il existe une fonction $h_{2, \varepsilon}$ telle que $\left(B(\varepsilon), h_{2, \varepsilon}^{2} g_{\text {euc }}\right)$ soit la réunion d'un cylindre de longueur $L$ et d'un calotte sphérique dont le bord s' appuie sur le bord du cylindre (voir figure 4). De plus, on peut choisir $h_{2, \varepsilon}$ de sorte que lorsque $\varepsilon$ tend vers zéro, la métrique de la sphère portant la calotte soit fixée. En appliquant cette déformation sur chacune des $k N$ boules euclidiennes contenues dans $\left(M, g_{\varepsilon}\right)$, la variété est isométrique à celle obtenue en attachant $k N$ sphères à $\left(M, g_{\varepsilon}\right)$ par des anses de rayon $\varepsilon$, on se trouve bien dans les condition d'application du théorème 3.1 .

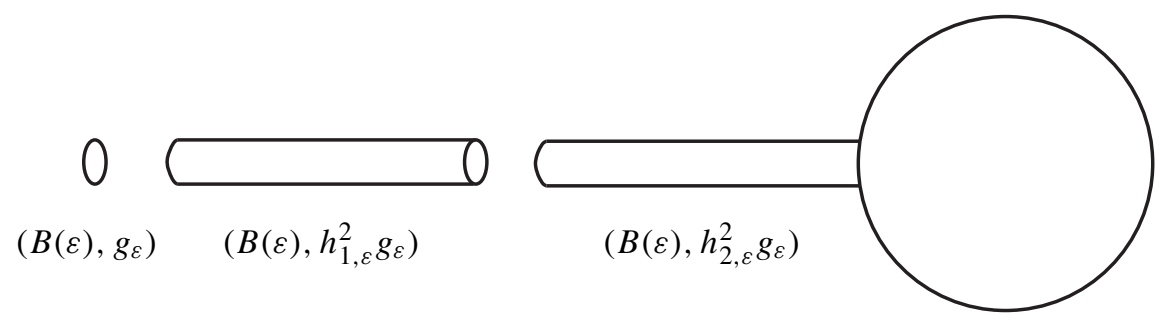

Figure 4

Il reste à transplanter sur chacune des sphères la métrique $g_{p_{0}, i}$ qu'on a défini précédemment. Pour cela, remarquons que dans la construction de la métrique $g_{p_{0}, i}$ à partir de la métrique canonique, il y a un ouvert $u$ de la sphère sur lequel la déformation conforme est une simple homothétie, dont on notera $\rho$ le rapport (voir remarque 2.4). On fixe un point $x \in \mathcal{U}$, et on identifie la calotte sphérique qu'on a construit à la calotte de $\left(S^{n}, \rho^{2} g_{\text {can }}\right)$ dont le bord est centré en $x$. On peut alors déformer la métrique sur la calotte de sorte qu'elle soit isométrique à la restriction de $g_{p_{0}, i}$. Si $\varepsilon$ est suffisamment petit, le bord de la calotte est entièrement contenu dans $\mathcal{U}$, il n'est donc pas déformé et la métrique globale est bien continue le long du bord.

En appliquant ces déformations sur chacune des $k N$ boules de $\left(M, g_{\varepsilon}\right)$, on construit une fonction $h_{3, \varepsilon}$ sur $M$ telle que $\left(M, h_{3, \varepsilon}^{2} g_{\varepsilon}\right)$ soit formé de $\left(M, g_{\varepsilon}\right)$ reliés aux $k N$ sphères $\left(S^{n}, g_{p_{0}, i}\right)$ par des anses dont le rayon tend vers zéro quand $\varepsilon$ tend vers zéro. Le théorème 3.1 assure alors que

$$
\mu_{p, i}\left(M, h_{3, \varepsilon}^{2} g_{\varepsilon}\right) \rightarrow \xi_{p, i} \text { quand } \varepsilon \rightarrow 0
$$

pour $1 \leq p \leq k$ et $1 \leq i \leq N$, les autres valeurs propres tendant vers des valeurs supérieures à $\sup _{p, i}\left\{v_{p, i}\right\}+\delta$ et le volume tendant vers $V$. 
La famille de métrique $h_{3, \varepsilon}^{2} g_{\varepsilon}$ n'est pas conforme à la métrique initiale $g$. Cependant, comme on a $\frac{1}{\tau(\varepsilon)} g_{\varepsilon} \leq g \leq \tau(\varepsilon) g_{\varepsilon}$, on a aussi

$$
\frac{1}{\tau(\varepsilon)} h_{3, \varepsilon}^{2} g_{\varepsilon} \leq h_{3, \varepsilon}^{2} g \leq \tau(\varepsilon) h_{3, \varepsilon}^{2} g_{\varepsilon}
$$

avec $\tau(\varepsilon) \rightarrow 1$. On peut en outre approcher la famille de fonction $\left(h_{3, \varepsilon}\right)_{\varepsilon}$ par une famille $\left(h_{4, \varepsilon}\right)_{\varepsilon}$ formée de fonctions lisses et telle que $e^{-\varepsilon}<h_{4, \varepsilon} / h_{3, \varepsilon}<e^{\varepsilon}$. La famille $\left(h_{4, \varepsilon} g\right)$ est alors constituée de métriques lisses conformes à $g$ et vérifie

$$
\frac{1}{e^{\varepsilon} \tau(\varepsilon)} h_{3, \varepsilon}^{2} g_{\varepsilon} \leq h_{4, \varepsilon}^{2} g \leq e^{\varepsilon} \tau(\varepsilon) h_{3, \varepsilon}^{2} g_{\varepsilon}
$$

Le lemme 2.1 assure alors que

$$
\lim _{\varepsilon \rightarrow 0} \mu_{p, i}\left(M, h_{4, \varepsilon}^{2} g\right)=\lim _{\varepsilon \rightarrow 0} \mu_{p, i}\left(M, h_{3, \varepsilon}^{2} g_{\varepsilon}\right)=\xi_{p, i}
$$

pour $1 \leq p \leq k$ et $1 \leq i \leq N$ et que les autres valeurs propres restent supérieures à $\sup _{p, i}\left\{v_{p, i}\right\}$ pour $\varepsilon$ suffisamment petit, et en outre $\operatorname{Vol}\left(M, h_{4, \varepsilon}^{2} g\right)$ tend vers $V$. Cette construction peut être réalisée pour n'importe quelle famille $\left(\xi_{p, i}\right)$ du domaine de la fonction $\Phi_{\varepsilon}$ définie en (3.4). On est donc en mesure d'appliquer le lemme de stabilité 3.3 et d'en déduire le théorème 1.3 .

\section{References}

[AC95] C. Anné et B. Colbois, Spectre du laplacien agissant sur les $p$-formes diférentielles et écrasement d'anses. Math. Ann. 303 (1995), 545-573. Zbl 0909.58054 MR 1355003

[Am03] B. Ammann, A spin-conformal lower bound of the first positive Dirac eigenvalue. Differerential Geom. Appl. 18 (2003), 21-32. Zbl 1030.58020 MR 1951070

[CdV86] Y. Colin de Verdière, Sur la multiplicité de la première valeur propre non nulle du laplacien. Comment. Math. Helv. 61 (1986), 254-270. Zbl 0607.53028 MR 0856089

[CdV87] Y. Colin de Verdière, Construction de laplaciens dont une partie finie du spectre est donnée. Ann. Sci. École Norm. Sup. 20 (1987), 599-615. Zbl 0636.58036 MR 0932800

[CES03] B. Colbois et A. El Soufi, Extremal eigenvalues of the Laplacian in a conformal class of metrics: the "conformal spectrum". Ann. Global Anal. Geom. 24 (2003), 337-349. Zbl 1036.58026 MR 2015867

[CES06] B. Colbois et A. El Soufi, Eigenvalues of the laplacian acting on $p$-forms and metric conformal deformations. Proc. Amer. Math. Soc. 134 (2006), 715-721. Zbl 1095.35013 MR 2180889

[Co04] B. Colbois, Spectre conforme et métriques extrémales. Sémin. Théor. Spectr. Géom. 22 (2004), 93-101. Zbl 1069.58017 MR 2136138 
[Da05] M. Dahl, Prescribing eigenvalues of the Dirac operator. Manuscripta Math. 118 (2005), 191-199. Zbl 1081.58021 MR 2177685

[Do82] J. Dodziuk, Eigenvalues of the Laplacian on forms. Proc. Amer. Math. Soc. 85 (1982), 437-443. Zbl 0502.58038 MR 0656119

[ESI86] A. El Soufi et S. Ilias, Immersions minimales, première valeur propre du laplacien et volume conforme. Math. Ann. 275 (1986), 257-267. Zbl 0675.53045 MR 0854009

[GP95] G. Gentile et V. Pagliara, Riemannian metrics with large first eigenvalue on forms of degree p. Proc. Amer. Math. Soc. 123 (1995), 3855-3858. Zbl 0848.53022 MR 1277111

[Gu04] P. Guérini, Prescription du spectre du laplacien de Hodge-de Rham. Ann. Sci. École Norm. Sup. 37 (2004), 270-303. Zbl 1068.58016 MR 2061782

[Ja04] P. Jammes, Petites valeurs propres des fibrés principaux en tores. Prépublication, 2004. arXiv:math.DG/0404536.

[Ja06] P. Jammes, Minoration conforme du spectre du laplacien de Hodge-de Rham. Manuscripta Math. 123 (2007), 15-23. Zbl 1127.35027MR 2300056

[Ko93] N. Korevaar, Upper bounds for eigenvalues of conformal metrics. J. Differerential Geom. 37 (1993), 73-93. Zbl 0794.58045 MR 1198600

[Lo86] J. Lott, Eigenvalue bounds for the Dirac operator. Pacific J. Math. 125 (1986), 117-126. Zbl 0605.58044 MR 0860754

[Lo96] J. Lohkamp, Discontinuity of geometric expansions. Comment. Math. Helv. 71 (1996), 213-228. Zbl 0857.58041 MR 1396673

[Mc93] J. McGowan, The $p$-spectrum of the Laplacian on compact hyperbolic three manifolds. Math. Ann. 279 (1993), 725-745. Zbl 0801.53034 MR 1245416

Received August 29, 2006

Pierre Jammes, Université d'Avignon, Laboratoire de Mathématiques, 33 rue Louis Pasteur, 84000 Avignon, France

E-mail: Pierre.Jammes@univ-avignon.fr 